\title{
HIGH-RESOLUTION TEMPORAL RECONSTRUCTION OF ANKLE JOINT FROM DYNAMIC MRI
}

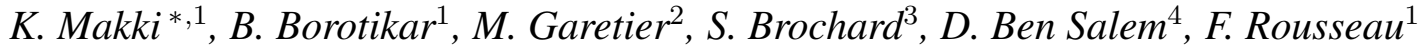 \\ ${ }^{1}$ Institut Mines Télécom Atlantique, LaTIM U1101 INSERM, UBL, Brest, France \\ ${ }^{2}$ Service d'Imagerie Médicale, HIA Brest, LaTIM U1101 INSERM, France \\ ${ }^{3}$ Rehabilitation Medicine department, CHRU Brest, LaTIM U1101 INSERM, France \\ ${ }^{4}$ Service d'Imagerie Médicale, Cavale Blanche, CHRU Brest, LaTIM U1101 INSERM, France
}

\begin{abstract}
Cerebral palsy is the leading cause of motor disabilities affecting children. The ankle is the most common equine musculoskeletal strain in children with cerebral palsy. Despite multiple medical and surgical therapies, postoperative recurrence rate is still very high (48\%). A major reason for therapy failure is the lack of knowledge of the ankle joint biomechanics. Dynamic MRI can be used to acquire high resolution static data and low resolution temporal images. However, spatial and temporal data should be combined to provide the most comprehensive point of view to study joint motion. In this paper, we first present an intensity-based registration method to accurately estimate the rigid motion of the ankle bones. Second, we investigate the use of the log-euclidean framework to reconstruct a four-dimensional (3D+time) high-resolution dynamic MRI sequence from a low-resolution dynamic sequence and one high resolution static MR image. The proposed approach has been applied and evaluated on in vivo MRI data acquired for a pilot study on child motor disability. Results demonstrate the robustness of the proposed pipeline and very promising high resolution visualization of the ankle joint.
\end{abstract}

Index Terms - High-resolution reconstruction, dynamic MRI, intensity-based registration, locally rigid transformation

\section{INTRODUCTION}

Cerebral palsy is the leading cause of child motor disabilities [1]. This is a term for permanent developmental disorders of movement and posture, responsible of activity limitations caused by nonprogressive damage occurring during early brain development. Approximately $90 \%$ of the deformities caused by cerebral palsy occur in the ankle and foot regions. The muscle contractions associated with the defect bone growth impact directly on the posture. These disorders are a frequent causative factor of moral distress for the patient and his/her family. Logistical and financial costs are thus added to the psychological cost.

Management of spastic equinus deformity is still a clinical challenge. There is a general agreement that surgical intervention is the most viable option for fixed equinus deformity with the goal to stabilize the lower extremity, and improve patient walk abilities. However, post-surgical statistics and the long term follow-up studies show

*. The research leading to these results has received funding from Région Bretagne, Chaire INSERM-IMT Atlantique, Fondation de l'Avenir, Fondation Motrice. up to $48 \%$ of recurrence rate and shows a general lack of understanding of spastic equinus deformity in children.

In this context, dynamic MRI is a non-invasive method that can provide spatial and temporal in vivo information of the joint [2]. Acquired MRI data usually consist in a high-resolution static scan containing very accurate anatomical information and a series of lowresolution dynamic scans capturing slow joint motion. However, the combined use of high resolution static data and temporal sequences is limited. Joint analysis of these in vivo data could provide major insights of biomechanical studies of human locomotion.

Joint analysis of high resolution static data and temporal sequences has been used by Gilles et al. in [3] to select of the best dynamic imaging protocol and to track bone motion from real-time dynamic MRI. A 2D/3D registration was used to estimate the hip joint kinematics of healthy volunteers. Clarke et al. have described in [4] a non-invasive 3D dynamic MRI method for measuring muscle moment arms in vivo. In this work, which is close to our study, the authors have proposed a 3D mesh-based registration method using an Iterative Closest Point (ICP) algorithm. Bone motions were estimated by the registration of high-resolution joint geometry from MRI scans of the stationary joint with low-resolution geometries from ultrafast MRI scans of the slowly moving joint. The main limitation is the need of manual segmentation of each low-resolution time-frame in addition to the segmentation of the high-resolution static scan.

In this article, we focus on the estimation of ankle joint dynamics of children from MRI. Contrary to the previous work of Clarke et al., we propose to use an automatic intensity-based registration technique to avoid the need of dynamic MRI segmentation. Moreover, we also investigate in this work the use of log-euclidean polyrigid framework developed by Arsigny et al. in [5] for ankle joint analysis. In [6], the authors prove that this type parameterization is well-adapted to the registration of articulated structures such as the hip and knee joint for serial x-ray CT mouse images. In [7], an extension of the Log Euclidean polyrigid registration was presented to deal with human articulated structures from 2D radiographs. In our study, we propose to use the log-euclidean framework to estimate high-resolution temporal MRI data. Using a set of bone-dependent rigid transforms, a dense temporal deformation field can be estimated by fusing multiple rigid transforms through the dynamic sequence. 


\section{CLINICAL DATA}

MRI data have been acquired using a 3T MR scanner (Achieva dStream, Philips Medical Systems, Best, NL). An MRI safe orthotic fixture has been specifically designed in such way that it can be used for performing either passive plantar-dorsiflexion or voluntary active ankle joint movements. These two cases will be called "passive motion" and "active motion" in the next sections. Data were acquired as part of a pilot study for children between 7 and 14 years old at CHRU of Brest, France, under a protocol approved by the local ethical committee. The scanning protocol included one highresolution static $3 \mathrm{D}$ scan of the stationary ankle joint with a resolution of $0.26 \times 0.26 \times 0.8 \mathrm{~mm}$, and three low-resolution dynamic scans (two passive sequences for two knee angle positions and one active sequence per child). Each dynamic sequence is composed of 15 time frames with a resolution of $0.57 \times 0.57 \times 8 \mathrm{~mm}$.

\section{METHOD}

The proposed approach for dynamic MRI analysis consists in two steps : 1) rigid motion estimation of each bone from dynamic sequence, 2) computation of dense deformation fields by fusing multiple rigid transforms.

\subsection{Motion estimation}

The first step of the proposed method is the motion estimation of each bone. In the context of ankle MRI, we focus on three bones : calcaneus, talus and tibia. In this work, bone motion is modeled with a $3 \mathrm{D}$ rigid transform (i.e. rotations and translations). The objective of this step is two-fold : 1) to estimate a mapping for each bone between high resolution static MRI data and dynamic sequences, 2) to estimate the relative motion of each bone using the dynamic sequence.

In this work, it is assumed that the bones of interest have been segmented in the high resolution static image (see Figure 1). In order to reduce manual interventions as much as possible, an intensitybased registration is used to propagate the initial segmentation from the high resolution static image through the dynamic low resolution sequence.

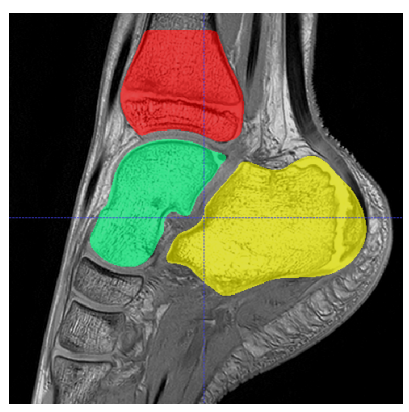

(a)

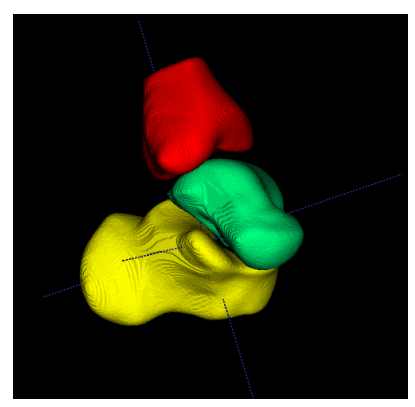

(b)
Fig. 1: Bones of interest : calcaneus (yellow), talus (green) and tibia (red). (a) : Mid-sagittal image from the high-resolution static scan; (b) : Three dimensional rendering of segmented bones.

The first step is to estimate the transformation from the highresolution static image to the dynamic sequence. This leads to the estimation of a rigid transformation for each bone between images that undergo non-rigid deformations (due to the movement of the joint). In order to make this step robust, the registration is carried out in two steps. First, a rigid registration between the static image and all the images in the dynamic sequence is performed. Then, initialized from the previous step, a rigid transformation is estimated between the static image and each image of the dynamic sequence for each bone. Finally, to maximize the robustness of the algorithm, a image from the dynamic sequence whose the content is the closest to the static image, is selected. To this end, the image of the sequence for which the overlap between regions of interest before and after bone-dependent registration is maximum is selected as the reference image for dynamic motion estimation.

The second step focuses on the estimation of the motion of each bone during the dynamic sequence. To do this, we propose to take advantage of the temporal regularity of the data by propagating the masks of each bone in the dynamic sequence. For each bone, a rigid transform is estimated between successive images. All the registrations are performed using FSL-FLIRT (FMRIB's Linear Image Registration Tool [8]) with correlation ratio as similarity measure. A voxel-wise weighting based on the segmentation masks is used when estimating bone-dependent motion.

The overall algorithm for bone motion estimation is described in Algorithm 1, using the following notations : $S$ is the high-resolution static image, $\left\{D_{k}\right\}_{k=1, \ldots, K}$ is the set of low resolution dynamic images, $\left\{M^{i}\right\}_{i=1, \ldots, N}$ is the set of mask of bones of interest, $T_{A \rightarrow B}$ is the rigid transform from image $A$ to image $B, T_{A \rightarrow B}^{i}$ is the rigid transform from image $A$ to image $B$ for the bone $i$, Dice $(\mathrm{A}, \mathrm{B})$ is the DICE coefficient which measures the overlap between segmented regions $\left(\operatorname{Dice}(A, B)=\frac{2|A \cap B|}{|A|+|B|}\right)$.

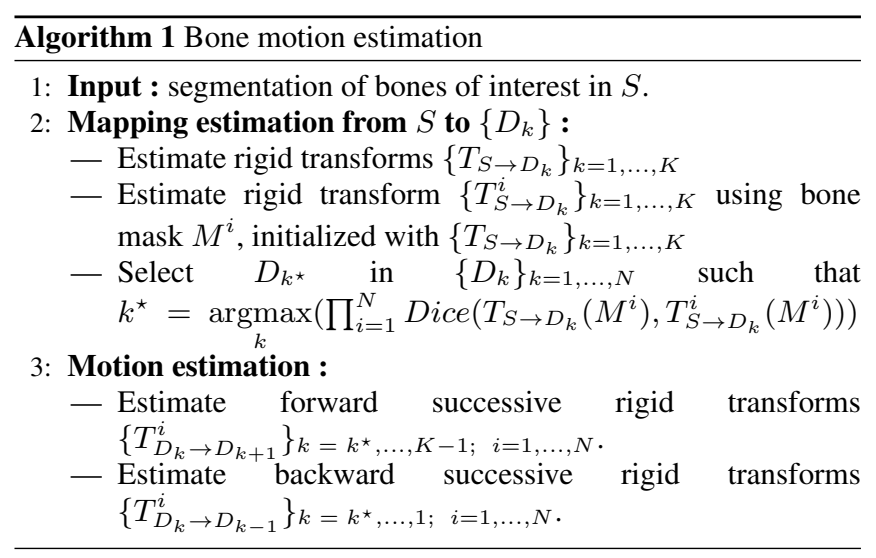

\subsection{Fusion of locally rigid transformations}

Given a set of rigid transforms for each bone at every time frame of the dynamic MRI sequence, a high resolution dynamic MRI sequence can be estimated by computing a set of dense deformation fields corresponding to each time frame. To this end, each dense deformation field is obtained by fusing rigid transforms corresponding to the bones of interest. For each bone, a weighting function is computed based on a distance to the mask [9]. Each weighting function reflects the local influence of the rigid transform of each bone of interest. In this study, following previous work of Commowick et al. [10], for each voxel $x$, the weighting functions are computed as follows :

$$
w_{D_{k}}^{i}(x)=\frac{1}{1+\alpha \operatorname{dist}\left(x, M_{D_{k}}^{i}\right)}
$$


where $\operatorname{dist}\left(x, M_{D_{k}}^{i}\right)$ is the distance between the point $x$ and the mask of the bone $i$ propagated onto the dynamic image $D_{k}, \alpha$ is set to 0.5 according to [10] to ensure smooth interpolation.

Given a point $x$ in the high-resolution static image, the target location of this point in the dynamic image $D_{k}$ can be computed within the log-euclidean polyrigid framework proposed by Arsigny et al. in [5], with the following equation :

$$
\mathcal{T}_{S \rightarrow D_{k}}(x)=\exp \left(\sum_{i=1}^{N} \tilde{w}_{D_{k}}^{i}(x) \log \left(T_{S \rightarrow D_{k}}^{i}\right)\right) \cdot x
$$

where $\mathcal{T}_{S \rightarrow D_{k}}$ is the dense deformation field from the static image $S$ to the dynamic image $D_{k}, \tilde{w}_{D_{k}}^{i}$ is a normalized weighting function (i.e. $\sum_{i=1}^{N} \tilde{w}_{D_{k}}^{i}(x)=1, \forall x \in D_{k}$ ). Figure 2 shows normalized weighting functions for the three bones of interest and the corresponding high-resolution estimated image.

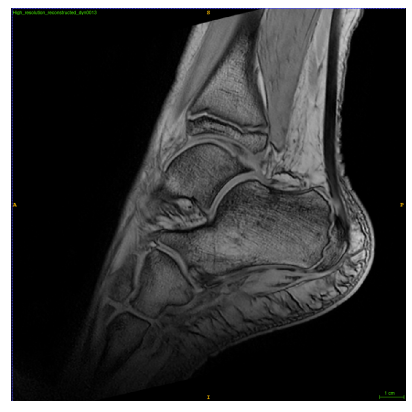

(a)

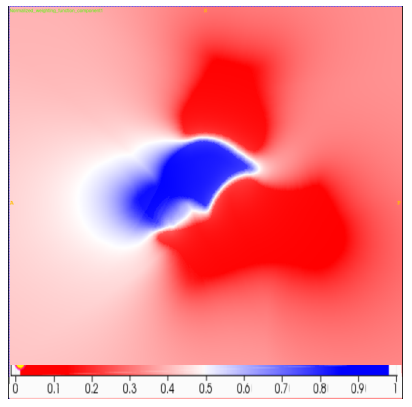

(c)

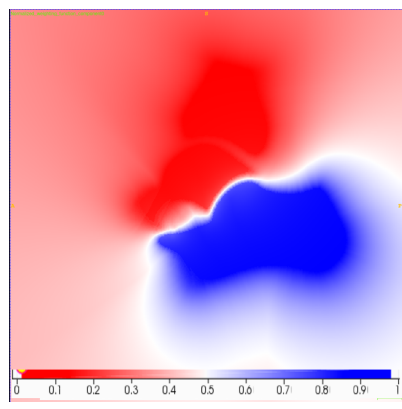

(b)

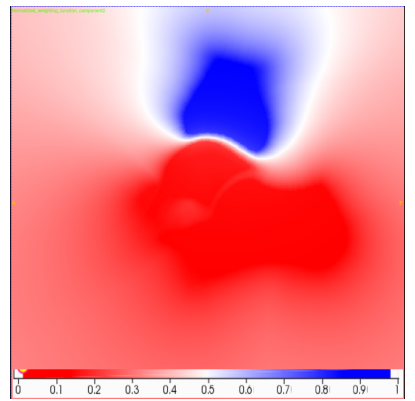

(d)
Fig. 2: Normalized weighting functions $\tilde{w}_{D_{k}}^{i}(x)$. (a) : corresponding high resolution reconstructed image, weighting function for the calcaneus (b), talus (c), tibia (d) with the following color coding : blue if greater than 0.5 , red if lesser than 0.5 , white if equal to 0.5 . The use of polyrigid framework leads to smooth diffeomorphic dense deformation fields.

\section{RESULTS}

Each high resolution static image of the dataset has been carefully manually segmented to extract accurate region of interest of the three bones considered in this work (calcaneus, talus and tibia, see Figure 1). In order to take into account the partial volume effect due to anisotropy of the image resolution, a Gaussian blur with a standard deviation set to 2 voxels has been applied onto each bone mask.
To evaluate the proposed registration approach for bone motion estimation, the propagated mask of each bone through the dynamic sequence is compared with a manually delineated bone on the first and the last dynamic image (i.e. $D_{1}$ and $D_{K}$ ). The accuracy of the estimations of the successive rigid transforms is assessed by computing the following DICE coefficient $\operatorname{Dice}\left(B_{k}^{i}, B_{m_{k}}^{i}\right)$ where $B_{k}^{i}$ is the propagated mask of the bone $i$ on dynamic image $D_{k}$ and $B_{m_{k}}^{i}$ is a manually delineated mask of the same bone on the dynamic mask $D_{k}$. A DICE value close to one indicates that the bone masks have been well propagated through the entire dynamic sequence.

Results on bone motion estimation are reported in Table 1 for six subjects of the pilot study. Results for passive and active motion are separated. In 33 of 36 cases, the DICE coefficient is greater than 0.8 indicating accurate propagations of bone masks over the dynamic sequence, for both passive and active motions. Although based on a successive estimation of transformations, the proposed approach appears to be robust enough for almost all subjects. It can be noticed that the proposed approach can be used for both passive and active motion of the ankle joint.

From the set of rigid transforms of each bone over the dynamic sequence, it is then possible to compute a temporal dense deformation field to synthesize a high resolution temporal image of the ankle joint. Figure 3 illustrates, for two subjects, such high resolution reconstructed data. The use of a diffeomorphic approach for polyrigid transform fusion leads to the estimation of realistic high resolution temporal data to study in vivo joint motion through dynamic MRI.

\section{CONCLUSION}

Our work focuses on dynamic MRI analysis for child ankle joint study. An intensity-based registration pipeline have been proposed to estimate bone motion through the dynamic sequence. The robustness of this approach for both passive and active motion has been evaluated on in vivo data acquired for a pilot study on child motor disability due to cerebral palsy. We have also investigated the use of log-euclidean framework to estimate dense temporal deformation fields from multiple rigid transforms. The polyrigid approach provides a way to combine high resolution spatial information with temporal dynamics of joints. Visual promising results have been presented showing the potential of this framework for detailed in vivo biomechanical analysis of joint motion from dynamic MRI.

In future works, the proposed approach will be applied to a larger dataset to compare ankle joint dynamics of children with cerebral palsy with age-matched healthy children. We will further explore this scheme to extract fine biomechanical parameters of tendon and ligaments. Dynamic MRI and dedicated image processing techniques can open a new way to study in vivo human joints.

\section{REFERENCES}

[1] A Colver, C Fairhurst, and P-OD Pharoah, "Cerebral palsy," The Lancet, vol. 383, no. 9924, pp. 1240-1249, 2014.

[2] Won C Bae, T Ruangchaijatuporn, and CB Chung, "New techniques in $\mathrm{mr}$ imaging of the ankle and foot," Magnetic Resonance Imaging Clinics, vol. 25, no. 1, pp. 211-225, 2017.

[3] B Gilles, R Perrin, N Magnenat-Thalmann, and J-P Vallee, "Bone motion analysis from dynamic MRI : acquisition and tracking," Academic Radiology, vol. 12, no. 10, pp. 12851292, 2005. 


\begin{tabular}{|c|c|c|c|c|c|c|c|c|c|c|c|c|}
\hline Passive motion & \multicolumn{2}{|c|}{ subject 1} & \multicolumn{2}{|c|}{ subject 2} & \multicolumn{2}{|c|}{ subject 3} & \multicolumn{2}{|c|}{ subject 4} & \multicolumn{2}{|c|}{ subject 5} & \multicolumn{2}{|c|}{ subject 6} \\
\hline \multirow[b]{2}{*}{ Calcaneus } & $k=1$ & $k=K$ & $k=1$ & $k=K$ & $k=1$ & $k=K$ & $k=1$ & $k=K$ & $k=1$ & $k=K$ & $k=1$ & $k=K$ \\
\hline & 0.95 & 0.97 & 0.96 & 0.92 & 0.96 & 0.96 & 0.98 & 0.95 & 0.97 & 0.98 & 0.99 & 0.99 \\
\hline Talus & 0.87 & 0.92 & 0.82 & 0.92 & 0.94 & 0.97 & 0.95 & 0.91 & 0.65 & 0.6 & 0.99 & 0.98 \\
\hline Tibia & 0.94 & 0.97 & 0.94 & 0.96 & 0.99 & 0.97 & 0.96 & 0.99 & 0.95 & 0.92 & 0.99 & 0.99 \\
\hline Active motion & \multicolumn{2}{|c|}{ subject 1} & \multicolumn{2}{|c|}{ subject 2} & \multicolumn{2}{|c|}{ subject 3} & \multicolumn{2}{|c|}{ subject 4} & \multicolumn{2}{|c|}{ subject 5} & \multicolumn{2}{|c|}{ subject 6} \\
\hline & $k=1$ & $k=K$ & $k=1$ & $k=K$ & $k=1$ & $k=K$ & $k=1$ & $k=K$ & $k=1$ & $k=K$ & $k=1$ & $k=K$ \\
\hline Calcaneus & 0.88 & 0.9 & 0.95 & 0.92 & 0.97 & 0.91 & 0.88 & 0.9 & 0.87 & 0.9 & 0.92 & 0.94 \\
\hline Talus & 0.8 & 0.88 & 0.9 & 0.9 & 0.94 & 0.92 & 0.93 & 0.95 & 0.52 & 0.45 & 0.4 & 0.99 \\
\hline Tibia & 0.95 & 0.98 & 0.96 & 0.9 & 0.97 & 0.97 & 0.99 & 0.95 & 0.95 & 0.96 & 0.98 & 0.97 \\
\hline
\end{tabular}

Table 1: 3D DICE coefficients between manual and automatic bone segmentation maps for the six subjects of the pilot study. $k=1$ corresponds to the first time frame of the dynamic sequence while $k=K$ corresponds to the last time frame.

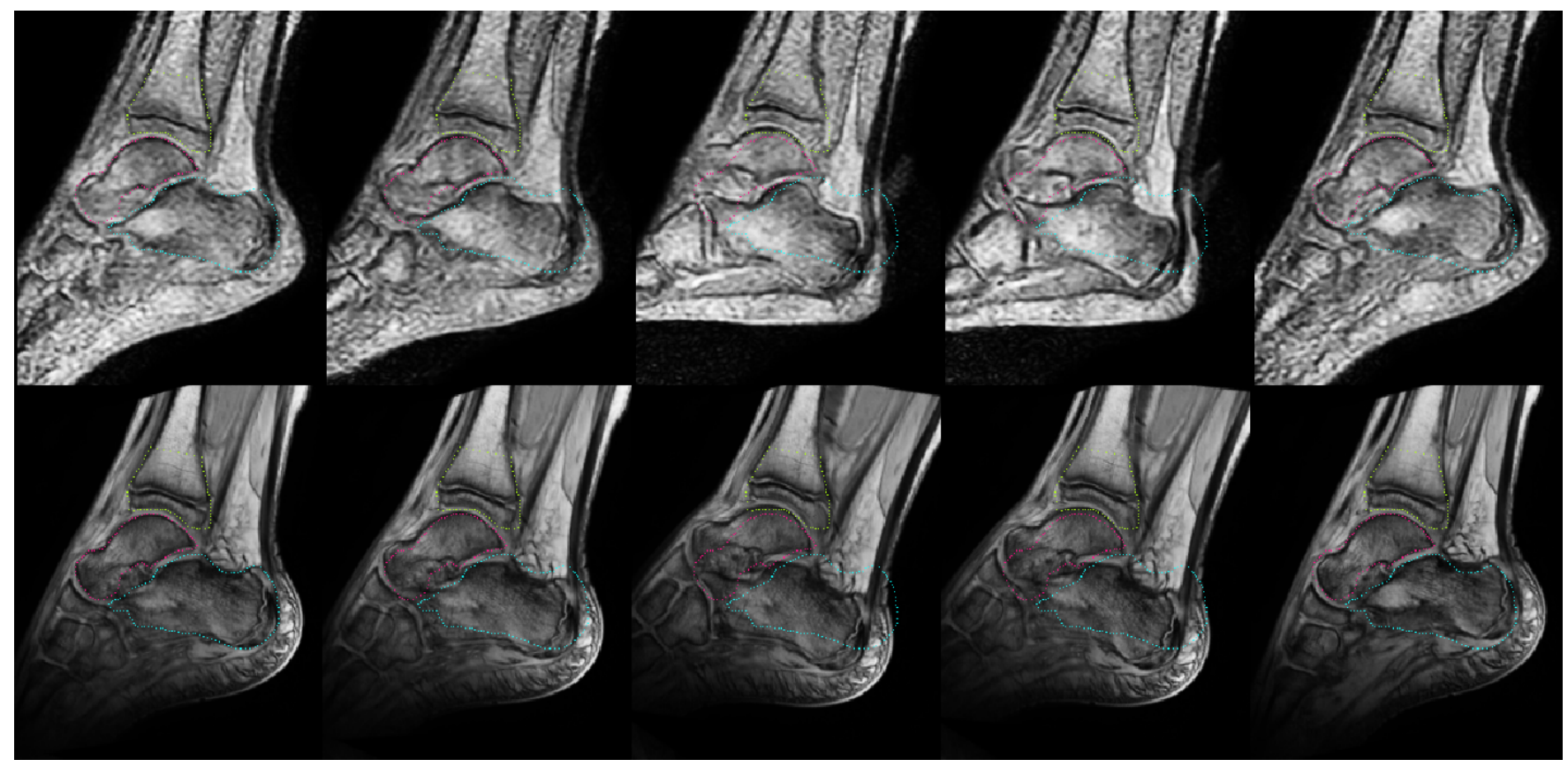

Fig. 3: High resolution temporal reconstruction for one subject (subject3). First row : original dynamic images (time frames : 1, 3, 6, 9 and 13) , second row : corresponding high resolution reconstructed image. Each column corresponds to one time frame. The first time frame contours shows the reconstruction accuracy (column 1) and the joint motion (columns 2 to 5)

[4] EC Clarke, JH Martin, AG d'Entremont, MG Pandy, DR Wilson, and RD Herbert, "A non-invasive, 3d, dynamic MRI method for measuring muscle moment arms in vivo : demonstration in the human ankle joint and achilles tendon," Medical Engineering \& Physics, vol. 37, no. 1, pp. 93-99, 2015.

[5] V Arsigny, X Pennec, and N Ayache, "Polyrigid and polyaffine transformations : a novel geometrical tool to deal with non-rigid deformations-application to the registration of histological slices," Medical Image Analysis, vol. 9, no. 6, pp. 507-523, 2005.

[6] X Papademetris, DP Dione, LW Dobrucki, LH Staib, and AJ Sinusas, "Articulated rigid registration for serial lowerlimb mouse imaging," in International Conference on Medical Image Computing and Computer-Assisted Intervention. Springer, 2005, pp. 919-926.

[7] MÁ Martín-Fernández, M Martín-Fernández, and C AlberolaLópez, "A log-euclidean polyaffine registration for articulated structures in medical images," in International Conference on Medical Image Computing and Computer-Assisted Intervention. Springer, 2009, pp. 156-164.

[8] M Jenkinson, P Bannister, M Brady, and S Smith, "Improved optimization for the robust and accurate linear registration and motion correction of brain images," Neuroimage, vol. 17, no. 2, pp. 825-841, 2002.

[9] V Arsigny, O Commowick, N Ayache, and X Pennec, "A fast and log-euclidean polyaffine framework for locally linear registration," Journal of Mathematical Imaging and Vision, vol. 33, no. 2, pp. 222-238, 2009.

[10] O Commowick, V Arsigny, A Isambert, J Costa, F Dhermain, F Bidault, P-Y Bondiau, N Ayache, and G Malandain, "An efficient locally affine framework for the smooth registration of anatomical structures," Medical Image Analysis, vol. 12, no. 4, pp. 427-441, 2008. 